\title{
Síndrome de apnea-hipopnea del sueño y enfermedad cardiovascular
}

\section{Sleep apnea-hypopnea syndrome and cardiovascular diseases}

\author{
B. Marín, B. Fernández, F. J. Domínguez
}

\section{RESUMEN}

El síndrome de apnea-hipopnea del sueño se caracteriza por un cuadro de somnolencia diurna excesiva, trastornos cognitivos-conductuales, respiratorios, cardíacos, metabólicos o inflamatorios secundarios a episodios repetidos de obstrucción de la vía aérea superior durante el sueño. Numerosos estudios han demostrado que el SAHS se asocia a la presencia de hipertensión arterial y al desarrollo de enfermedades cardiovasculares. Asimismo se acepta un exceso de mortalidad asociado con el SAHS. Como mecanismos mediadores se consideran un aumento de actividad del sistema nervioso simpático, una disfunción endotelial, junto con fenómenos de estrés oxidativo, agregación plaquetaria y trombosis.

Palabras clave. Apnea del sueño. Enfermedad cardiovascular. Hipertensión arterial.

An. Sist. Sanit. Navar. 2007; 30 (Supl. 1): 89-95.

\begin{abstract}
Sleep apnea-hypopnea syndrome is characterised by daytime sleepiness, cognitive-behavioural, respiratory, cardiac, metabolic or inflammatory disorders, secondary to repeated obstruction of the upper airway during sleep. Numerous studies have shown that SAHS is associated with the presence of arterial hypertension and the development of cardiovascular diseases. Similarly, there is an excess of mortality associated with SAHS. An increase in the activity of the sympathetic nervous system and endothelial dysfunction are considered as mediating mechanisms, together with phenomena of oxidative stress, platelet aggregation and thrombosis.
\end{abstract}

Key words. Sleep apnea. Cardiovascular diseases. Arterial hypertension.
Sección de Neumología. Hospital de Navarra. Pamplona.

\section{Correspondencia:}

Belén Marín Martínez

Sección de Neumología

Hospital de Navarra

Irunlarrea, 3

31008 Pamplona

Tfno. 848422288

E-mail:0 bmarinma@cfnavarra.es 


\section{INTRODUCCIÓN}

El síndrome de apneas-hipopneas del sueño (SAHS) consiste en la aparición de episodios recurrentes de limitación del paso del aire durante el sueño como consecuencia de una alteración anatómicofuncional de la vía aérea superior (VAS) que conduce a su colapso provocando descensos de la saturación de oxihemoglobina $\left(\mathrm{SaO}_{2}\right)$ y microdespertares que dan lugar a un sueño no reparador, somnolencia diurna excesiva, trastornos neuropsiquiátricos, respiratorios y cardíacos $^{1}$. El Grupo Español de Sueño (GES) en su Consenso Nacional sobre el síndrome de apneas-hipopneas del sueño define recientemente éste como un cuadro de somnolencia excesiva, trastornos cognitivos-conductuales, respiratorios, cardíacos, metabólicos o inflamatorios secundarios a episodios repetidos de obstrucción de la vía aérea superior durante el sueño. Estos episodios se miden con el índice de alteración respiratoria (IAR), considerado como la suma del IAH (índice de apneahipopnea por hora de sueño) y los esfuerzos respiratorios asociados a los microdespertares (ERAM). Un IAR $>5$ asociado con síntomas relacionados con la enfermedad y no explicados por otras causas confirma el diagnóstico. El IAR se define por el número de apneas, hipopneas y ERAM por hora de sueño².

El SAHS es una enfermedad muy prevalente que afecta al 4-6\% de los varones y al $2-4 \%$ de las mujeres en la población general adulta de edades medias ${ }^{3,4}$. Numerosos estudios han demostrado que el SAHS está asociado al deterioro de la calidad de vida, presencia de hipertensión arterial, desarrollo de enfermedades cardiovasculares y cerebrovasculares y relacionado con la aparición de accidentes de tráfico. Asimismo se acepta un exceso de mortalidad asociada con el SAHS.

\section{FISIOPATOLOGÍA}

Los episodios apneicos nocturnos originan fenómenos de hipoxemia, reoxigenación, hipercapnia, cambios en la presión intratorácica y fragmentación del sueño que ocasionan una vasoconstricción sistémica y pulmonar con hipertensión arterial pulmonar y sistémica secundaria, así como aumento de la poscarga y reducción del gasto cardíaco. Como mecanismos mediadores se consideran un aumento de actividad del sistema nervioso simpático, una disfunción endotelial con desequilibrio entre factores vasoconstrictores $\mathrm{y}$ vasodilatadores, junto con fenómenos de estrés oxidativo (consecuencia de las repetidas desaturaciones y reoxigenaciones), agregación plaquetaria y trombosis ${ }^{5,6}$.

El SAHS se ha asociado a alteraciones en el metabolismo de proteínas, hormonas y citoquinas que intervienen en el control del peso corporal, la regulación del sueño, la inflamación y el riesgo cardiovascular. Se ha observado en estos pacientes aumento de las concentraciones plasmáticas de interleucina-6 (IL6) y una alteración en el ritmo circadiano de secreción de factor de necrosis tumoral, ambos inducen la síntesis de proteína $\mathrm{C}$ reactiva, que constituye un marcador de inflamación y un factor de riesgo independiente de patología cardiovascular?.

\section{SAHS E HIPERTENSIÓN ARTERIAL}

Se ha estimado que entre un $40 \mathrm{y}$ un $60 \%$ de los pacientes con SAHS son hipertensos y aproximadamente un tercio de los pacientes con hipertensión arterial (HTA) padecen un SAHS. Pero esta relación se ha cuestionado porque los pacientes con estas enfermedades comparten ciertas características que pueden actuar como factores de confusión (sexo, edad, obesidad, consumo de alcohol y tabaco entre otros). En los pacientes con SAHS se producen aumentos cíclicos de la tensión arterial en relación con los episodios obstructivos que acontecen durante la noche. En esto se hallan implicados los quimiorreceptores centrales y periféricos, barorreceptores y aferentes pulmonares, la hipoxia y la hipercapnia, los aumentos en la presión negativa intratorácica y los despertares transitorios, dando lugar a una serie de cambios autonómicos, hemodinámicos y humorales que ocasionan un efecto presor al finalizar las apneas. El debate radica en si estas alteraciones durante la noche pueden provocar HTA sostenida durante el día ${ }^{8}$. 
Estudios epidemiológicos realizados sobre grandes muestras han demostrado una asociación entre el IAH y la HTA.

Lavie y $\mathrm{col}^{9}$ en una muestra de 2.677 pacientes de edades entre 20 y 85 años, demuestran que la tensión arterial y el número de pacientes hipertensos aumentan linealmente con la gravedad del IAH. Por cada aumento del IAH en una unidad, el riesgo relativo para HTA se incrementaba en $1 \%$, mientras que cada $10 \%$ de descenso en la saturación de oxígeno nocturna incrementaba el riesgo un 13\%, concluyendo que el SAHS esta asociado con HTA independientemente de otros factores de riesgo. Asimismo Young y col $^{10}$ en una muestra de 1060 trabajadores de edades comprendidas entre los 30 y 60 años observaron que la prevalencia de HTA aumentaba en relación con la gravedad del IAH independientemente de la edad, sexo e índice de masa corporal.

En el estudio de Wisconsin ${ }^{11}$ se realiza un seguimiento prospectivo de 709 personas durante 4 años objetivándose una relación dosis-respuesta entre el IAH basal y la presencia de HTA encontrada 4 años después, independientemente de otros factores de riesgo, sugiriendo causalidad. El estudio demuestra que incluso elevaciones mínimas del IAH se asocian con un aumento de la odds ratio (OR) en el desarrollo de una HTA tras 4 años de seguimiento. Las odds ratios para la presencia de HTA fueron 1,42 (IC 95\% 1,13$1,78), 2,03(1,29-3,17)$ y $2,89(1,46-5,64)$ con IAH menor de 5; entre 5-15 y mayor de 15 eventos/hora respectivamente.

Los resultados del Sleep Heart Health Study $y^{12}$, estudio trasversal multiétnico que incluye a 6.132 personas mayores de 40 años de ambos sexos demuestra una asociación entre el SAHS y la HTA independientemente de los factores confusionales y proporcional a la gravedad del SAHS, así la presencia de un índice de apneas-hipopneas de 30 por hora se acompañaba de una odds ratio de HTA de 1,37, cuando se comparaba con los sujetos con un IAH menor de 1,5 por hora. Recientemente se ha sugerido que la contribución de la apnea obstructiva del sueño al riesgo de hipertensión puede ser sexo-dependiente y mayor en varones que en mujeres ${ }^{13}$.
Por tanto se puede asumir que el SAHS es un factor de riesgo independientemente de HTA. En 1997 el Sexto informe del Comité Nacional Americano para la Prevención, Detección, Evaluación y Tratamiento de la Hipertensión Arterial (HTA) reconoció por primera vez la importancia de descartar el SAHS como un factor que contribuye a la HTA resistente y más recientemente en su Séptimo Informe de 2003, este Comité ha incluido el SAHS como la primera en la lista de causas identificables de $\mathrm{HTA}^{14,15}$. Por tanto el SAHS siempre debe considerarse en el diagnóstico diferencial de la HTA refractaria y la HTA de reciente diagnóstico, especialmente en pacientes obesos hipertensos y en pacientes cuya presión arterial no se reduce en el período nocturno (no dippers) ${ }^{16}$.

Se ha estudiado también el papel del tratamiento con presión continua positiva por vía nasal (CPAP) en la disminución de la tensión arterial en pacientes con SAHS, existiendo varios trabajos aleatorizados y controlados que evalúan este efecto. Pepperell y col${ }^{17}$ estudiaron 118 pacientes con SAHS que recibieron CPAP en dos grupos (presión óptima y presión subterapéutica). El grupo con presión óptima redujo su presión arterial (PA) media 2,5 mmHg mientras que el grupo con presión subterapéutica aumento la presión media $0,8 \mathrm{mmHg}$ ( $\mathrm{p}=0,0013)$. El beneficio se observó en la PA sistólica y diastólica, tanto por el día como por la noche. El beneficio fue mayor en los pacientes con SAHS más severos y en sujetos diagnosticados de HTA. Faccenda y col $^{18}$ también demostraron en un estudio con 68 pacientes reducciones en las cifras de PA media diastólica y sistólica, siendo el descenso de la PA mayor en aquellos pacientes que desaturaban más durante el sueño.

Mae y col ${ }^{19}$, observaron que en pacientes hipertensos sin tratamiento farmacológico, tres semanas de tratamiento con CPAP reducía la PA nocturna sistólica y diastólica de forma significativa comparada con una CPAP de tipo placebo. Becker y $\mathrm{col}^{20}$ mostraron en 32 pacientes con SAHS severo que nueve semanas de tratamiento con CPAP eficaz reducían la PA diurna y nocturna en alrededor de $10 \mathrm{mmHg}$ mientras que no hubo variaciones en un grupo 
de pacientes comparables tratados con una CPAP subterapéutica. Logan y $\mathrm{col}^{21}$ en un ensayo terapéutico no controlado en pacientes con HTA refractaria, observaron que la utilización de CPAP se acompañó de una disminución de $11 \pm 4,4 \mathrm{mmHg}$ de la PA sistólica de 24 horas. La PA diastólica nocturna también disminuyó significativamente $(7,8 \pm 3 \mathrm{mmHg})$.

Por otra parte, Barbé y $\mathrm{col}^{22}$ han observado que el tratamiento con CPAP no parece producir beneficios en términos de PA, calidad de vida y pruebas cognitivas a corto plazo (6 semanas) en pacientes paucisintomáticos. Robinson y $\mathrm{col}^{23}$ randomizaron a 35 pacientes con SAHS, hipertensos sin somnolencia a recibir CPAP terapéutica o subterapéutica durante 4 semanas no encontrando diferencias significativas en la presión media de 24 horas, concluyendo que en pacientes con SAHS no somnolientos la CPAP no reduce las cifras de TA.

\section{SAHS Y CARDIOPATÍA ISQUÉMICA}

El SAHS se ha relacionado con una mayor incidencia de enfermedad arteriosclerótica coronaria. La hipoxia y, su principal consecuencia cardiovascular, la hipertensión sistémica aumentan, a través de un incremento en la disfunción endotelial, el riesgo de enfermedad coronaria en los SAHS graves. El SAHS debe considerarse como un factor de riesgo más que debe ser tratado en pacientes con enfermedad coronaria y sospecha clínica. Igualmente se debe considerar la presencia clínica de enfermedad coronaria en pacientes con SAHS con mayor motivo que en la población general al evaluar los factores de riesgo coronario ${ }^{2}$.

Diversos estudios han demostrado la asociación entre los trastornos respiratorios durante el sueño y la enfermedad de las arterias coronarias. Schäfer y $\mathrm{col}^{24}$ encuentran una alta prevalencia de SAHS en pacientes con enfermedad coronaria angiográficamente demostrada, concluyendo que la existencia de un SAHS moderado $(\mathrm{IAH}>20)$ se asoció con una OR de tener un infarto de miocardio de 2 (IC 1,0$3,8)$. Shahar y $\mathrm{col}^{25}$ en el estudio epidemiológico Sleep Heart Health Study, demues- tran una asociación entre los trastornos respiratorios del sueño y los episodios de isquemia coronaria (OR 1,27, IC 0,99-1,62).

En pacientes con enfermedad arterial coronaria establecida, el SAHS severo puede desencadenar isquemia nocturna cardiaca con depresión del segmento ST (predominantemente en fase de movimientos oculares rápidos) a menudo resistente al tratamiento habitual ${ }^{26,27}$. Milleron y $\mathrm{col}^{28}$, en un estudio prospectivo de 54 pacientes con cardiopatía isquémica y SAHS observan que el tratamiento del SAHS está asociado a una reducción del riesgo de muerte cardiovascular.

\section{SAHS Y ARRITMIAS}

El SAHS se ha asociado con arritmias rápidas auriculares en relación con un incremento en la presión telediastólica ventricular. En el SAHS esto se puede encontrar en pacientes con una presión auricular izquierda elevada por presentar, tanto como factor coadyuvante del SAHS o como efecto, un grado avanzado de miocardiopatía ventricular izquierda. La existencia de cor pulmonale subsiguiente a la hipertensión arterial pulmonar también se relacionará con la presencia de taquiarritmias auriculares. El tratamiento específico de esta enfermedad será fundamentalmente el control de la situación desencadenante. En cuanto a la incidencia de bradiarritmias, sobre todo nocturnas, en el SAHS es muy alta. Son en su mayoría arritmias neuromediadas a través de un intenso reflejo vagal cardioinhibidor que se han relacionado de forma directa con las desaturaciones nocturnas. En resumen, la presencia de taquiarritmias en pacientes con SAHS debe obligarnos a investigar la existencia de otras enfermedades desencadenantes y dirigir el tratamiento hacia su control. Asimismo la presencia de bradiarritmias en los pacientes con SAHS no es una indicación de estimulación cardiaca permanente en la actualidad. Los pacientes con indicación de estimulación cardiaca que presenten un SAHS podrían beneficiarse de la estimulación nocturna a frecuencias superiores que su frecuencia media basal, en especial si se registran mayoritariamente apneas centrales ${ }^{2}$. 
Mehra y $\operatorname{col}^{29}$ comparan la prevalencia de arritmias en dos muestras de participantes del Sleep Heart Health Study, demostrando que independientemente de otros factores de confusión, las personas con SAHS tienen mayor riesgo de tener fibrilación auricular OR 4,02 (IC 95\% 1,03-15,74); taquicardia ventricular no sostenida OR 3,40 (IC 95\% 1,03-11,20) y extrasístoles ventriculares OR 1,74 (IC $95 \%$ 1,11-2,74).

\section{SAHS E INSUFICIENCIA CARDIACA}

Se ha observado una relación entre el SAHS grave y la miocardiopatía dilatada idiopática. Las alteraciones de oxigenación y sus consecuencias vasculares y sobre la poscarga pueden constituir un factor de riesgo definitivo en la aparición de afección miocárdica y en la progresión de la insuficiencia cardíaca congestiva. Los pacientes con miocardiopatía dilatada con criterios clínicos de sospecha de SAHS deben estudiarse mediante poligrafía nocturna ya que su enfermedad cardíaca puede responder positivamente al tratamiento de su SAHS ${ }^{2}$.

Los estudios epidemiológicos demuestran una asociación entre el SAHS y la insuficiencia cardiaca. De este modo un análisis de la cohorte del Sleep Heart Health Study demuestra que el SAHS es un factor de riesgo de desarrollo de insuficiencia cardíaca independientemente de otros factores de riesgo OR 2,38 $(1,22-4,62)^{25}$. Asimismo en pacientes con insuficiencia cardíaca tratada farmacológicamente y SAHS el tratamiento añadido con CPAP permite reducir la presión arterial sistólica y mejorar la función sistólica del ventrículo izquierdo ${ }^{30}$.

Arias y $\mathrm{col}^{31}$ han demostrado que el SAHS puede asociarse a una disfunción ventricular diastólica del ventrículo izquierdo independientemente de otros factores. La aplicación de CPAP durante 3 meses puede evitar la progresión de estas alteraciones incluso revertirlas al menos en estadios iniciales antes del desarrollo de cambios estructurales. Alonso-Fernández y col $^{32}$ observan que los pacientes con SAHS normotensos y función ventricular izquierda conservada ya presentan una peor respuesta cardíaca al ejercicio que sujetos sanos que también mejora después del tratamiento con CPAP.

\section{SAHS Y MORTALIDAD}

La evidencia acumulada sugiere que existe un exceso de mortalidad asociado al SAHS no tratado. Marín y $\mathrm{col}^{33}$ siguieron durante 10 años a un grupo de 264 varones sanos, 377 roncadores simples, 403 con SAHS leve-moderado no tratados, 235 con SAHS severo no tratados y 372 tratados con CPAP. Los pacientes con enfermedad severa no tratada tuvieron una mayor incidencia de eventos cardiovasculares fatales (1,06 x 100 personas y año) y eventos cardiovasculares no fatales $(2,13 \times 100$ personas y año) con respecto a pacientes con SAHS leve-moderado no tratados, roncadores simples, pacientes tratados con CPAP y participantes sanos. El análisis multivariante para factores confusores mostró que el SAHS severo no tratado aumenta significativamente el riesgo de eventos cardiovasculares fatales (OR 2,87, 95\% IC $1,17-7,51$ ) y no fatales (OR 3,17, 95\% IC $1,12-7,51)$, comparado con los participantes sanos. El tratamiento con CPAP reduce este riesgo.

Gami y $\mathrm{col}^{34}$ estudiaron las polisomnografías y los certificados de defunción de 112 pacientes y encontraron que los sujetos con IAH>40 tenían una mayor mortalidad durante las horas de sueño en comparación con los sujetos sin SAHS y que esta mortalidad se debía a causas cardíacas.

Campos-Rodríguez y col ${ }^{35}$ realizaron un seguimiento durante 4 años a 871 pacientes diagnosticados de SAHS tratados con CPAP. De los 871 pacientes 46 murieron durante el seguimiento. La principal causa de muerte fueron las enfermedades cardiovasculares. Se distribuyeron los pacientes en tres categorías según el número de horas de uso de CPAP (menos de 1 hora, entre 1-6 horas/día y más de 6 horas/día) siendo la mortalidad mayor entre los pacientes que no recibieron tratamiento con CPAP (menos de 1hora/día).

La asociación entre SAHS y enfermedad cerebrovascular se expone en otro capítulo. 


\section{CONCLUSIÓN}

En resumen, numerosos estudios demuestran que el SAHS se asocia a la presencia de hipertensión arterial y al desarrollo de enfermedades cardiovasculares, por lo tanto siempre debe considerarse en el diagnóstico diferencial de la HTA refractaria e HTA de reciente diagnóstico. Asimismo ante un paciente con un episodio cardiovascular agudo debe investigarse en la anamnesis la existencia de un SAHS y su posible papel como un factor agravante y/o desencadenante del episodio. También debe ser considerado el diagnóstico de SAHS en pacientes con insuficiencia cardíaca refractaria, isquemia cardiaca nocturna y arritmias nocturnas, especialmente en personas con factores de riesgo para apnea del sueño.

\section{BIBLIOGRAFÍA}

1. Montserrat JM, Amilibia J, Barbé F, Capote F, DurÁn J, Mangado NG et al. Tratamiento del síndrome de las apneas-hipopneas durante el sueño. Arch Bronconeumol 1998; 34: 204206.

2. Grupo Español de Sueño. Consenso Nacional sobre el síndrome de apneashipopneas del sueño. Arch Bronconeumol 2005; 41, Supl 4: 3S-110S.

3. Young T, Palta M, Dempsey J, Skatrud J, Weber $\mathrm{S}$, BADR S. The occurrence of sleep disorders breathing among middle aged adults. N Engl J Med 1993; 328: 1230-1236.

4. Durán J, Esnaola S, Ramón R, IzTUeta A. Obstructive sleep apnea-hypopnea and related clinical features in a populationbased sample of subjects aged 30 to 70 years. Am J Respir Crit Care Med 2001; 163 : 685-689.

5. Shamsuzzaman AS, Gersh BJ, Somers VK. Obstructive sleep apnea. Implications for Cardiac and Vascular Disease JAMA 2003; 290: 1906-1914.

6. LeUnG R, BRADlEy D. Sleep apnea and cardiovascular disease. Am J Respir Crit Care Med 2001; 164: 2147-2165.

7. JimÉnez A, Aguirregomoscorta I, Barceló A, Chiner E, PAyo F. Fisiopatología, genética y biología molecular del síndrome de apneashipopneas del sueño. Arch Bronconeumol 2002; 38 (Supl 3): 10-14.

8. Terán J, Del Campo F, Mayoralas LR, Villasante C, Parra O, Hernández L. Calidad de vida. Riesgo cardiovascular y cerebrovascular. Accidentalidad y mortalidad. Arch Bronconeumol 2002; 38(Supl 3): 15-20.

9. LAVIE P, Herer P, Hoffstein V. Obstructive sleep apnea syndrome as a risk factor for hypertension: population study. BMJ 2000; 320: 479-482.

10. Young T, Peppard P, Palta M, Mae K, Finn L, Morgan B et al. Population-Based Study of Sleep-Disordered Breathing as a risk factor for hypertension. Arch Intern Med 1997; 157: 1746-1752.

11. Peppard P, Young T, Palta M, Skatrud J. Prospective study of the association between sleep-disordered breathing and hypertension. N Engl J Med. 2000; 342: 13781384.

12. Nieto FJ, Young T, Lind B, Shahar E, Samet J, REDLINE $\mathrm{S}$ et al. Association of sleepdisordered breathing, sleep apnea and hypertension in a large community-based study. JAMA 2000; 283: 1829-1836.

13. Hedner J, Bengtsson-Boström K, Peker Y, Grote L, RASTAM L, LindBLAd U. Hypertension prevalence in obstructive sleep apnea and sex: a population-based case-control study. Eur Respir J 2006; 27: 564-570.

14. The sixth report of the Joint National Committee on Prevention, Detection, Evaluation and Treatment of High Blood Pressure. Arch Intern Med 1997; 157: 24132446.

15. Chobanian AV, BaKris GL, Black HR, Cushman W, GReEn L, Izzo J. The Seventh Report of the Joint National Committee on Prevention, Detection, Evaluation and Treatment of High Blood Pressure: the JNC 7 report. JAMA 2003; 289:2560-2572.

16. Wolk R, Somers VK. Cardiovascular consequences of obstructive sleep apnea. Clin Chest Med 2003; 24: 195-205.

17. Pepperell J, Ramdassingh-Dow S, Crosthwaite N, Mullins R, Jenkinson C, Stradling J et al. Ambulatory blood pressure after therapeutic and subtherapeutic nasal continuous positive airway pressure for obstructive sleep apnea: a randomised parallel trial. Lancet 2001; 359: 204-210.

18. Faccenda J, Mackay T, Boon N, Douglas N. Randomized placebo-controlled trial of continuous positive airway pressure on blood pressure in the sleep apnea-hipopnea syndrome. Am J Respir Crit Care Med 2001; 163: 344-348.

19. Mae K, Skatrud J, Finn L, Palta M, Young T. The effect of correction of sleep-disordered 
breathing on BP in untreated hypertension. Chest 2002; 122: 1125-1132.

20. Becker H, Jerrentrup A, Ploch T, Psych D, Grote L, Penzel $\mathrm{T}$ et al. Effect of nasal continuous positive airway pressure treatment on blood pressure in patients with obstructive sleep apnea. Circulation 2003; 107: 68-73.

21. Logan AG, Tkacova R, Perlikowski SM, Leung RS, TISLER A, FlORAS JS. Refractory hypertension and sleep apnoea: effect of CPAP on blood pressure and baroreflex. Eur Respir J 2003; 21: 241-247.

22. Barbé F, Mayoralas LR, Durán J, Masa JF, Maimo A, Montserrat JM. Treatment with continuous positive airway pressure is not effective in patients with sleep apnea but no daytime sleepiness. Ann Inter Med 2001; 134: 1015-1023.

23. Robinson GV, Smith DM, LANGFord BA, Davies RJ, StRAdLING JR. Continuous positive airway pressure does not reduce blood pressure in nonsleepy hypertensive OSA patients. Eur Respir Dis 2006; 27: 1229-1235.

24. SchäFER H, Koehler U, EwIG S, HaSper E, TASCI S, LÜDERITZ B. Obstructive sleep apnea as a risk marker in coronary artery disease. Cardiology 1999; 92: 79-84.

25. Shahar E, Whitney C, Redine S, Lee S, Newman A, NIETo FJ et al. Sleep-disordered breathing and cardiovascular disease. Am Respir Crit Care Med. 2001; 163: 19-25.

26. Franklin K, Nilsson J, SAHLin C, Näslund U. Sleep apnoea and nocturnal angina. Lancet 1995; 345: 1085-1087.

27. Mooe T, Franklin K, WikLund U, RabBen T, Holmström K. Sleep-disordered breathing and myocardial ischemia in patiens with coronary artery disease. Chest 2000; 117 : 1597-1602.

28. Milleron O, Pillière R, Foucher A, DE Roquefeuil F, Aegerter P, Jondeau G et al.
Beneficts of obstructive sleep apnoea treatment in coronary artery disease: a longterm follow-up study. Eur Heart J 2004; 25: 728-734.

29. Mehra R, Benjamin EJ, Shahar E, Gottlieb DJ, NAWABIT R, KIRCHNER HL et al. Association of nocturnal arrhythmias with sleepdisordered breathing: The Sleep Heart Health Study. Am J Respir Crit Care Med 2006; 173: 910-916

30. Kaneko Y, Floras J, Phil D, Usui K, Plante J, TKACOVA R et al. Cardiovascular effects of continuous positive airway pressure in patients with heart failure and obstructive sleep apnea. N Engl J Med 2003; 348: 12331241.

31. Arias M, García Río F, Alonso-Fernández A, Mediano O, Martínez I, Villamor J. Obstructive sleep apnea syndrome affects left ventricular diastolic function. Circulation 2005; 112: 375-383.

32. Alonso-Fernández A, García Río F, Arias M, Mediano O, Pino JM, Martínez I et al. Obstructive sleep apnoea-hipoapnoea syndrome reversibly depresses cardiac response to exercise. Eur Heart J 2006; 27 : 207-215.

33. Marín JM, Carrizo SJ, Vicente E, Agustí A. Long-term cardiovascular outcomes in men with obstructive sleep apnoea-hipopnoea with or without treatment with continuous positive airway pressure: an observational study. Lancet 2005; 365: 1046-1053.

34. Gami A, Howard D, Olson E, Somers V. Daynight pattern of sudden death in obstructive sleep apnea. N England J Med 2005; 352:1206-1214.

35. Campos-Rodríguez F, Pena-Grinan N, ReyesNuÑez N, De la Cruz-Morón I, Perez-Ronchel J, DE LA VEGA-Gallardo. Mortality in obstructive sleep apnea-hipopnea patients treated with positive airway pressure. Chest 2005; 128: 264-233. 
